\title{
A Mathematical Analysis of the Strip-Element Method for the Computation of Dispersion Curves of Guided Waves in Anisotropic Layered Media
}

\author{
F. Schöpfer, F. Binder, A. Wöstehoff, and T. Schuster \\ Fakultät für Maschinenbau, Helmut-Schmidt-Universität, Holstenhofweg 85, 22043 Hamburg, Germany \\ Correspondence should be addressed to F. Schöpfer, schoepfer@hsu-hh.de
}

Received 24 February 2010; Accepted 4 June 2010

Academic Editor: Paulo Batista Gonçalves

Copyright (C) 2010 F. Schöpfer et al. This is an open access article distributed under the Creative Commons Attribution License, which permits unrestricted use, distribution, and reproduction in any medium, provided the original work is properly cited.

Dispersion curves of elastic guided waves in plates can be efficiently computed by the StripElement Method. This method is based on a finite-element discretization in the thickness direction of the plate and leads to an eigenvalue problem relating frequencies to wavenumbers of the wave modes. In this paper we present a rigorous mathematical background of the Strip-Element Method for anisotropic media including a thorough analysis of the corresponding infinite-dimensional eigenvalue problem as well as a proof of the existence of eigenvalues.

\section{Introduction}

In recent years there has been considerable interest in the study of the behaviour of elastic guided waves in plates due to their potential use in Nondestructive Evaluation (NDE) and Structural Health Monitoring (SHM); see, for example, the comprehensive books of Giurgiutiu [1] or Rose [2]. Already in isotropic plates Lamb waves are dispersive and the dispersion relations expressed by the Rayleigh-Lamb equations must be computed numerically; see, the study by Achenbach in [3]. Elastic wave propagation in layered and anisotropic media is an even more complex problem, and efficient numerical methods are required to obtain dispersion curves. Among those methods are the Transfer Matrix Method and the Global Matrix Method and we refer to the study by Lowe in [4] for an overview. One of the most efficient and flexible methods is based on a finite-element discretization in the thickness direction of the plate and leads to a generalized eigenvalue problem relating frequencies to wavenumbers of the Lamb wave modes. This method is known, amongst others, as the strip-element method (SEM) or layer-element method or semianalytic finiteelement-method; see, for example, the early works of Dong and Nelson [5] and Aalami [6], 
or Kausel [7], Galán and Abascal [8], the excellent book of Liu and Xi [9], and the many references therein. Also the works of Gavrić [10], Bartoli et al. [11], Marzani et al. [12], and Treyssède [13] could be of interest.

So far, there seems to be no strict mathematical analysis of the underlying infinitedimensional eigenvalue problem in the anisotropic case. For the isotropic case we recommend reading the paper by Bouhennache in [14], who also uses a more abstract setting. Since the SEM has been successfully applied in practice for several years, we think that it is worthwhile to start such an analysis and give here a mathematical proof of the existence of eigenvalues.

In the next section we recall some basic facts about generalized eigenvalue problems in Hilbert spaces. The differential equation governing the wave propagation in laminated plates is formulated in Section 3. In the main Section 4 we analyse the weak form of those equations for the elementary Lamb wave modes, show that weak and strong solutions coincide and are layerwise $\mathcal{C}^{\infty}$, and prove existence of weak solutions and eigenvalues of the related eigenvalue problem. Finally we present some numerical results illustrating the increasingly direction-dependent behaviour of transversely isotropic material with an increasing degree of anisotropy.

\section{Generalized Eigenvalue Problems in Hilbert Spaces}

Although the results of this section are wellknown, they might not always be explicitly found in the given form and therefore we prove some of them for the convenience of the reader. For further reading we suggest the books of Lax [15] and Conway [16].

Let $\mathscr{H}, \mathscr{H}_{1}, \mathscr{H}_{2}$ be real or complex Hilbert spaces with scalar products $\langle\cdot \mid \cdot\rangle$. By $\mathcal{L}\left(\mathscr{L}_{1}, \mathscr{H}_{2}\right)$ we denote the space of continuous linear operators from $\mathscr{H}_{1}$ to $\mathscr{L}_{2}$ and set $\mathcal{L}(\mathscr{\ell}):=\mathcal{L}(\mathscr{L}, \mathscr{L})$. For $T \in \mathcal{L}\left(\mathscr{l}_{1}, \mathscr{l}_{2}\right)$ we denote by $T^{*} \in \mathcal{L}\left(\mathscr{\ell}_{2}, \mathscr{\ell}_{1}\right)$ the adjoint operator defined by

$$
\langle T v \mid u\rangle_{\mathscr{L}_{2}}=\left\langle v \mid T^{*} u\right\rangle_{\mathscr{H}_{1}}, \quad \forall v \in \mathscr{L}_{1}, u \in \mathscr{H}_{2} .
$$

Range and nullspace are denoted by $\mathcal{R}(T)$ and $\mathcal{N}(T)$, respectively. We say that $T \in \mathcal{L}(\mathscr{\ell})$ is self-adjoint if $T^{*}=T$, and positive if

$$
\langle u \mid T u\rangle_{\mathscr{H}} \geq 0, \quad \forall u \in \mathscr{\ell} .
$$

Lemma 2.1. Let $T \in \mathcal{L}(\mathscr{\ell})$ be self-adjoint. If $T$ is injective and has closed range, then it is bijective and hence continuously invertible.

Proof. For $\mathcal{X} \subset \mathscr{L}$, let $\mathcal{X}^{\perp}=\{x \in \mathscr{H} \mid\langle x \mid y\rangle=0$, for all $y \in \mathscr{H}\}$ be the orthogonal complement of $\mathcal{X}$. The assertion follows from

$$
\mathcal{R}(T)=\overline{\mathcal{R}(T)}=\mathcal{N}\left(T^{*}\right)^{\perp}=\mathcal{N}(T)^{\perp}=\{0\}^{\perp}=\mathscr{H},
$$

which means that $T$ is also surjective. 
Lemma 2.2. An operator $T \in \mathcal{L}\left(\mathfrak{h}_{1}, \mathfrak{h}_{2}\right)$ is injective and has closed range if and only if there exists some constant $c>0$ such that

$$
\|T u\|_{\mathscr{H}_{2}} \geq c\|u\|_{\mathscr{H}_{1}}, \quad \forall u \in \mathscr{H}_{1} .
$$

Proof. See, for example, the study by Schröder in [17].

We obtain the following.

Corollary 2.3. An operator $T \in \mathcal{L}(\mathscr{\ell})$ is injective and has closed range if there exists some constant $c>0$ such that

$$
\langle u \mid T u\rangle_{\mathscr{H}} \geq c\|u\|_{\mathscr{H}}^{2}, \quad \forall u \in \mathscr{H} .
$$

Proof. Together with the Cauchy-Schwartz inequality, we obtain from (2.5)

$$
\|u\|_{\mathscr{L}}\|T u\|_{\mathscr{H}} \geq\langle u \mid T u\rangle_{\mathscr{H}} \geq c\|u\|_{\mathscr{L}}^{2}, \quad \forall u \in \mathscr{H} .
$$

With Lemma 2.2, we can conclude the assertion.

For $M, S \in \mathcal{L}(\mathscr{\ell})$, consider the generalized eigenvalue problem

$$
M u=\lambda S u .
$$

That is, "for which $\lambda \in \mathbb{R}, \mathbb{C}$ do nontrivial solutions $u \in \mathscr{\ell}$ to (2.7) exist?" In case $S$ is bijective, (2.7) is equivalent to the standard eigenvalue problem

$$
S^{-1} M u=\lambda u,
$$

and we readily obtain the following proposition which we state for the case $\operatorname{dim} \mathscr{t}=\infty$. It applies accordingly to the finite-dimensional case with finite sequences $\lambda_{n}, u_{n}$.

Proposition 2.4. Let $M, S \in \mathcal{L}(\mathscr{\ell})$ be self-adjoint and positive. Assume that $M$ is compact and injective and that $S$ is bijective. Then there exists a decreasing sequence $0<\lambda_{n} \rightarrow 0$ (counted with multiplicity) and a sequence $u_{n} \in \mathscr{d}$ such that

$$
\begin{aligned}
M u_{n} & =\lambda_{n} S u_{n} \\
\frac{1}{\lambda_{n}}\left\langle u_{n} \mid M u_{m}\right\rangle_{\mathscr{H}} & =\delta_{n, m}=\left\langle u_{n} \mid S u_{m}\right\rangle_{\mathscr{H}}
\end{aligned}
$$

and each $u \in \mathscr{L}$ can be uniquely represented by an $\mathfrak{d}$-convergent series

$$
u=\sum_{n=1}^{\infty} \alpha_{n} u_{n}
$$


with an $\ell_{2}$-sequence $\alpha_{n}=\left\langle u \mid S u_{n}\right\rangle_{\mathscr{H}}$. Especially, $\langle v \mid u\rangle_{S}:=\langle v \mid S u\rangle_{\mathscr{H}}$ defines a scalar product on $\mathscr{H}$ which induces an equivalent norm on $\mathscr{d e}$, and $u_{n}$ is an orthonormal basis with respect to this scalar product.

Proof. Since $S$ is self-adjoint, bijective, and positive, $\langle v \mid u\rangle_{S}:=\langle v \mid S u\rangle_{\mathscr{H}}$ indeed defines a scalar product on $\mathscr{H}$ which induces an equivalent norm on $\mathscr{H}$. With respect to this scalar product, the injective and compact operator $\widetilde{M}:=S^{-1} M$ is self-adjoint and positive. Therefore (2.9) is equivalent to the standard eigenvalue problem $\widetilde{M} u_{n}=\lambda_{n} u_{n}$ for the injective, compact, self-adjoint, and positive operator $\widetilde{M} \in \mathcal{L}\left\{\mathscr{\ell},\langle\cdot \mid \cdot\rangle_{S}\right\}$.

\section{Differential Equations in Matrix Notation}

We recall some relations of elasticity theory in matrix notation which is especially suited for the finite-element formulation [9]. For a profound study of the mathematical theory of elasticity in tensor notation, we refer to the book of Marsden and Hughes in [18].

(i) Differential operator matrix $L$ is given as,

$$
L=L_{x} \partial_{x}+L_{y} \partial_{y}+L_{z} \partial_{z}
$$

with the constant matrices

$$
L_{x}=\left(\begin{array}{ccc}
1 & 0 & 0 \\
0 & 0 & 0 \\
0 & 0 & 0 \\
0 & 0 & 0 \\
0 & 0 & 1 \\
0 & 1 & 0
\end{array}\right), \quad L_{y}=\left(\begin{array}{ccc}
0 & 0 & 0 \\
0 & 1 & 0 \\
0 & 0 & 0 \\
0 & 0 & 1 \\
0 & 0 & 0 \\
1 & 0 & 0
\end{array}\right), \quad L_{z}=\left(\begin{array}{lll}
0 & 0 & 0 \\
0 & 0 & 0 \\
0 & 0 & 1 \\
0 & 1 & 0 \\
1 & 0 & 0 \\
0 & 0 & 0
\end{array}\right) .
$$

(ii) Displacement vector $u$ is given as

$$
u=u(x, y, z, t)=\left(u_{x}, u_{y}, u_{z}\right)^{T} .
$$

(iii) Strain vector $\epsilon$ is given as

$$
\epsilon=\left(\epsilon_{x}, \epsilon_{y}, \epsilon_{z}, \gamma_{y z}, \gamma_{x z}, \gamma_{x y}\right)^{T}
$$

(iv) Strain-displacement relation is given as

$$
\epsilon=L u \text {. }
$$

(v) Stress vector $\sigma$ is given as

$$
\sigma=\left(\sigma_{x}, \sigma_{y}, \sigma_{z}, \tau_{y z}, \tau_{x z}, \tau_{x y}\right)^{T} .
$$




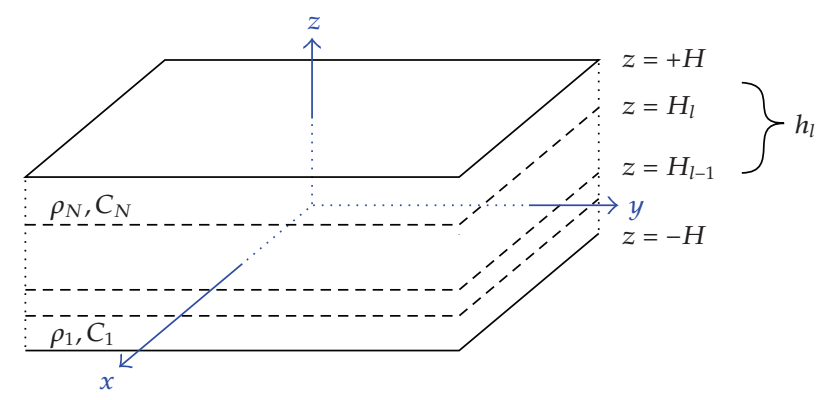

Figure 1: Laminate and coordinate systems.

(vi) And the generalized Hooke Law is given as

$$
\sigma=C \epsilon,
$$

with $C=\left(C_{i j}\right)_{i, j=1, \ldots, 6}$ being the matrix of material constants. In the following, $C$ is supposed to be real, symmetric, and positive definite (spd). For an isotropic material we have, for example,

$$
C_{\text {isotropic }}=\left(\begin{array}{cccccc}
\lambda+2 \mu & \lambda & \lambda & 0 & 0 & 0 \\
\lambda & \lambda+2 \mu & \lambda & 0 & 0 & 0 \\
\lambda & \lambda & \lambda+2 \mu & 0 & 0 & 0 \\
0 & 0 & 0 & \mu & 0 & 0 \\
0 & 0 & 0 & 0 & \mu & 0 \\
0 & 0 & 0 & 0 & 0 & \mu
\end{array}\right)
$$

with Lamé's constants $\lambda, \mu$.

Now consider a laminated plate of thickness $2 H$ in direction of the $z$-axis (middle $z=0$, top $z=+H$, bottom $z=-H$ ) and infinite in the $x$-y plane; see Figure 1 . The plate consists of $N$ layers. Layer $l=1, \ldots, N$ has thickness $h_{l}=H_{l}-H_{l-1}$ with $-H=H_{0}<H_{1}<$ $\cdots<H_{N}=+H$ and is supposed to consist of homogenous, anisotropic, elastic material with density $\rho_{l}$ and material constants $C_{l}$.

Let $u=\left(u_{x}, u_{y}, u_{z}\right)^{T}=u(x, y, z, t)$ be the displacement vector of a wave travelling in the plate in the absence of external forces. In each layer the elastic wave equation in matrix notation is

$$
\rho_{l} \ddot{u}=L^{T} C_{l} L u, \quad \forall x, y, t \text { and all } z \in\left(H_{l-1}, H_{l}\right) .
$$

The traction-free boundary conditions on the top and bottom surfaces are

$$
\left.L_{z}^{T} C_{N} L u\right|_{z=+H}=\left.L_{z}^{T} C_{1} L u\right|_{z=-H}=0 .
$$


Besides continuity of the displacement vector, the following interface conditions concerning continuity of the stresses are supposed to hold:

$$
\left.L_{z}^{T} C_{l} L u\right|_{z=H_{l}}=\left.L_{z}^{T} C_{l+1} L u\right|_{z=H_{l}}, \quad \forall l=1, \ldots, N-1 .
$$

As ansatz for a wave mode, we take a plane harmonic wave travelling in the $x$-y plane

$$
u(x, y, z, t)=\widehat{u}(z) e^{i\left(k_{x} x+k_{y} y \pm \omega t\right)}
$$

with $z$-dependent amplitude vector $\widehat{u}(z)=\left(\widehat{u}_{x}(z), \widehat{u}_{y}(z), \widehat{u}_{z}(z)\right)^{T}$, real circular frequency $\omega$, and real wave vector $k=\left(k_{x}, k_{y}\right)^{T}$. For such a wave mode, the wave equation (3.9), boundary (3.10) and interface conditions (3.11) reduce to

$$
\begin{gathered}
-\rho_{l} \omega^{2} \widehat{u}=L_{k}^{T} C_{l} L_{k} \widehat{u}, \quad \forall z \in\left(H_{l-1}, H_{l}\right), l=1, \ldots, N, \\
\left.L_{z}^{T} C_{N} L_{k} \widehat{u}\right|_{z=+H}=\left.L_{z}^{T} C_{1} L_{k} \widehat{u}\right|_{z=-H}=0, \\
\left.L_{z}^{T} C_{l} L_{k} \widehat{u}\right|_{z=H_{l}}=\left.L_{z}^{T} C_{l+1} L_{k} \widehat{u}\right|_{z=H_{l}}, \quad \forall l=1, \ldots, N-1,
\end{gathered}
$$

with the differential operator matrix $L_{k}$ given as

$$
L_{k}=i k_{x} L_{x}+i k_{y} L_{y}+L_{z} \partial_{z}
$$

Obviously for complex conjugation we have

$$
\bar{L}_{k}=-i k_{x} L_{x}-i k_{y} L_{y}+L_{z} \partial_{z}
$$

The question is "for which combinations of circular frequencies $\omega$ and wave vectors $k$ do nontrivial solutions $\widehat{u}$ of (3.13), (3.14), and (3.15) exist?" The answer leads to the dispersion relations $\omega(k)$ and is given in the next section. For better readability we will write $\omega$ instead of $\omega(k)$ in the following.

\section{Weak Form of the Reduced Wave Equation and Related Eigenvalue Problem}

We define the piecewise constant functions of density $\rho$ and matrix of material constants $C$ as

$$
\rho(z)=\rho_{l}, \quad C(z)=C_{l}, \quad \forall z \in\left(H_{l-1}, H_{l}\right), l=1, \ldots, N
$$

For suitable virtual displacements $\widehat{v}=\widehat{v}(z)$, which we define later, the weak form of (3.13), (3.14), and (3.15) can then be written as

$$
\omega^{2} \int_{-H}^{+H} \overline{\widehat{v}}^{T} \rho \widehat{u} d z=\int_{-H}^{+H}\left(\overline{L_{k} \widehat{v}}\right)^{T} C\left(L_{k} \widehat{u}\right) d z
$$


The next proposition shows that strong and weak solutions coincide for smooth enough functions.

Proposition 4.1. Suppose that $\widehat{u}=\widehat{u}(z)$ is continuous and layerwise $\mathcal{C}^{2}$; that is, $\widehat{u} \in \mathcal{C}([-H,+H])$ and $\left.\widehat{u}\right|_{\left(H_{l-1}, H_{l}\right)} \in \mathcal{C}^{2}\left(\left[H_{l-1}, H_{l}\right]\right), l=1, \ldots, N$. Then $\widehat{u}$ fulfills $(4.2)$ for all $\widehat{v} \in \mathcal{C}^{1}([-H,+H])$ if and only if $\widehat{u}$ is a solution of (3.13), (3.14), and (3.15).

Proof. Note that we shortly write $\left.\widehat{u}\right|_{\left(H_{l-1}, H_{l}\right)} \in \mathcal{C}^{2}\left(\left[H_{l-1}, H_{l}\right]\right)$ meaning that there is a function $\tilde{u} \in \mathcal{C}^{2}\left(\left[H_{l-1}, H_{l}\right]\right)$ such that $\left.\widehat{u}\right|_{\left(H_{l-1}, H_{l}\right)}=\left.\tilde{u}\right|_{\left(H_{l-1}, H_{l}\right)}$. By the smoothness assumptions on $\widehat{u}$, relation (3.17), and layerwise partial integration on the right-hand side of (4.2), we see that for all $\widehat{v} \in \mathcal{C}^{1}([-H,+H])(4.2)$ is equivalent to

$$
\omega^{2} \int_{-H}^{+H} \widehat{\widehat{v}}^{T} \rho \widehat{u} d z=-\int_{-H}^{+H} \overline{\widehat{v}}^{T} L_{k}^{T} C L_{k} \widehat{u} d z+\sum_{l=1}^{N}\left[\overline{\widehat{v}}^{T} L_{z}^{T} C_{l} L_{k} \widehat{u}\right]_{H_{l-1}}^{H_{l}}
$$

Let $\widehat{u}$ fulfill this equation for all $\widehat{v} \in \mathcal{C}^{1}([-H,+H])$. At first we fix a layer $l=1, \ldots, N$ and choose arbitrary functions $\widehat{v}$ that have compact support in the interior $\left(H_{l-1}, H_{l}\right)$ of the layer. For such functions, (4.3) reduces to

$$
\omega^{2} \int_{H_{l-1}}^{H_{l}} \overline{\widehat{v}}^{T} \rho_{l} \widehat{u} d z=-\int_{H_{l-1}}^{H_{l}} \overline{\widehat{v}}^{T} L_{k}^{T} C_{l} L_{k} \widehat{u} d z
$$

from which we infer that $\widehat{u}$ fulfills (3.13). Repeating this for all layers, we conclude that the integrals on the left- and right-hand side of (4.3) coincide and hence (4.3) reduces to

$$
0=\sum_{l=1}^{N}\left[\overline{\widehat{v}}^{T} L_{z}^{T} C_{l} L_{k} \widehat{u}\right]_{H_{l-1}}^{H_{l}} .
$$

Now by successively choosing functions $\widehat{v}$ that equal 1 in a vicinity of one of the points $-H=$ $H_{0}, H_{1}, \ldots, H_{N}=+H$ and that are 0 everywhere else, we see that $\widehat{u}$ also fulfills (3.14) and (3.15).

The converse assertion is obvious.

To prove existence of nontrivial weak solutions, we will transform (4.2) into a generalized eigenvalue problem in a suitable Hilbert space. Let $H^{1}=H^{1}(-H,+H)$ be the Sobolev space of all complex-valued square integrable functions on $(-H,+H)$ whose distributional first derivative can also be identified with a square integrable function; see, the study by Adams in [19]. For simplicity in the following we use the same symbol $H^{1}$ also for $\left(H^{1}\right)^{3}=H^{1} \times H^{1} \times H^{1},\left(H^{1}\right)^{6}, \ldots$, and likewise for other spaces like the spaces of square integrable functions $L^{2}=L^{2}(-H,+H)$ and continuously differentiable functions 
$\mathcal{C}^{1}=\mathcal{C}^{1}([-H,+H]), \ldots$, since it will become clear from the context how many components the vector-valued functions have. Endowed with the scalar product

$$
\begin{aligned}
& \langle\widehat{v} \mid \widehat{u}\rangle_{H^{1}}=\langle\widehat{v} \mid \widehat{u}\rangle_{L^{2}}+\left\langle\partial_{z} \widehat{v} \mid \partial_{z} \widehat{u}\right\rangle_{L^{2}} \\
& =\int_{-H}^{+H} \overline{\widehat{v}(z)}^{T} \widehat{u}(z) d z+\int_{-H}^{+H}{\overline{\partial_{z} \widehat{v}(z)}}^{T} \partial_{z} \widehat{u}(z) d z,
\end{aligned}
$$

the space $H^{1}$ is a complex Hilbert space. The space $\mathcal{C}^{1}$ is continuously embedded and dense in $H^{1}$ and the inclusions $H^{1} \hookrightarrow \mathcal{C}$ and $J: H^{1} \hookrightarrow L^{2}$ are compact. See, for example, the study by Maz'ja in [20]. Multiplications with the piecewise constant function $\rho>0$ and the piecewise constant spd-matrix $C$, respectively, define bijective, positive, self-adjoint, continuous linear operators on the respective $L^{2}$-spaces. Furthermore, the differential operator matrix $L_{k}$ (3.16) defines a continuous linear operator $L_{k}: H^{1} \rightarrow L^{2}$. For functions $\widehat{u}, \widehat{v} \in H^{1}$, which we assume in the following, the left-hand side of (4.2) can then be written as

$$
\omega^{2} \int_{-H}^{+H} \overline{\widehat{v}}^{T} \rho \widehat{u} d z=\omega^{2}\langle J \widehat{v} \mid \rho J \widehat{u}\rangle_{L^{2}}=\omega^{2}\left\langle\widehat{v} \mid J^{*} \rho J \widehat{u}\right\rangle_{H^{1}}=\omega^{2}\langle\widehat{v} \mid M \widehat{u}\rangle_{H^{1}}
$$

with the compact, injective, positive, and self-adjoint operator $M:=J^{*} \rho J$, and the right-hand side can be written as

$$
\int_{-H}^{+H}\left(\overline{L_{k} \widehat{v}}\right)^{T} C\left(L_{k} \widehat{u}\right) d z=\left\langle L_{k} \widehat{v} \mid C L_{k} \widehat{u}\right\rangle_{L^{2}}=\left\langle\widehat{v} \mid L_{k}^{*} C L_{k} \widehat{u}\right\rangle_{H^{1}}=\left\langle\widehat{v} \mid S_{k} \widehat{u}\right\rangle_{H^{1}}
$$

with the positive and self-adjoint operator $S_{k}:=L_{k}^{*} C L_{k}$. Thus, a function $\widehat{u} \in H^{1}$ fulfills (4.2) for all $\widehat{v} \in H^{1}$ if and only if

$$
\omega^{2}\langle\widehat{v} \mid M \widehat{u}\rangle_{H^{1}}=\left\langle\widehat{v} \mid S_{k} \widehat{u}\right\rangle_{H^{1}}, \quad \forall \widehat{v} \in H^{1},
$$

which is equivalent to the generalized eigenvalue problem

$$
\omega^{2} M \widehat{u}=S_{k} \widehat{u} \Longleftrightarrow\left(\gamma+\omega^{2}\right) M \widehat{u}=\left(\gamma M+S_{k}\right) \widehat{u} \Longleftrightarrow \lambda M \widehat{u}=S_{\gamma} \widehat{u}
$$

with $\lambda:=\gamma+\omega^{2}$ and the positive and self-adjoint operator $S_{\gamma}:=\gamma M+S_{k}$ for an arbitrary $\gamma>0$. We will need this disturbance with $\gamma M$ to prove that the operator $S_{\gamma}$ is also bijective and hence can apply Proposition 2.4 to guarantee nontrivial solutions. By Lemma 2.1 and Corollary 2.3, bijectivity of $S_{\gamma}$ follows by showing that there exists some constant $c>0$ such that

$$
\left\langle\widehat{u} \mid S_{\gamma} \widehat{u}\right\rangle_{H^{1}} \geq c\|\widehat{u}\|_{H^{1}}^{2}, \quad \forall \widehat{u} \in H^{1} .
$$


To show this, we write

$$
\begin{aligned}
\left\langle\widehat{u} \mid S_{\gamma} \widehat{u}\right\rangle_{H^{1}} & =\gamma\langle\widehat{u} \mid M \widehat{u}\rangle_{H^{1}}+\left\langle\widehat{u} \mid S_{k} \widehat{u}\right\rangle_{H^{1}} \\
& =\int_{-H}^{+H} \gamma \overline{\widehat{u}}^{T} \rho \widehat{u}+\left(\overline{L_{k} \widehat{u}}\right)^{T} C\left(L_{k} \widehat{u}\right) d z .
\end{aligned}
$$

Since layerwise $C$ is an spd-matrix and $\rho>0$, there is a constant $c_{1}>0$ such that pointwise a.e.

$$
\gamma \overline{\widehat{u}}^{T} \rho \widehat{u}+\left(\overline{L_{k} \widehat{u}}\right)^{T} C\left(L_{k} \widehat{u}\right) \geq c_{1}\left(\gamma \overline{\widehat{u}}^{T} \widehat{u}+\left(\overline{L_{k} \widehat{u}}\right)^{T}\left(L_{k} \widehat{u}\right)\right),
$$

and thus we have

$$
\left\langle\widehat{u} \mid S_{\gamma} \widehat{u}\right\rangle_{H^{1}} \geq c_{1} \int_{-H}^{+H} \gamma \overline{\hat{u}}^{T} \widehat{u}+\left(\overline{L_{k} \widehat{u}}\right)^{T}\left(L_{k} \widehat{u}\right) d z=c_{1} \int_{-H}^{+H} \overline{\tilde{u}}^{T} Q_{k} \tilde{u} d z
$$

with

$$
\tilde{u}=\left(\widehat{u}_{x}, \widehat{u}_{y}, \widehat{u}_{z}, \partial_{z} \widehat{u}_{x}, \partial_{z} \widehat{u}_{y}, \partial_{z} \widehat{u}_{z}\right)^{T}
$$

and the Hermitian matrix

$$
Q_{k}:=\left(\begin{array}{cccccc}
|k|^{2}+\gamma & k_{x} k_{y} & 0 & 0 & 0 & 0 \\
k_{x} k_{y} & |k|^{2}+\gamma & 0 & 0 & 0 & 0 \\
0 & 0 & |k|^{2}+\gamma & -i k_{x} & -i k_{y} & 0 \\
0 & 0 & i k_{x} & 1 & 0 & 0 \\
0 & 0 & i k_{y} & 0 & 1 & 0 \\
0 & 0 & 0 & 0 & 0 & 1
\end{array}\right)
$$

where $|k|^{2}=k_{x}^{2}+k_{y}^{2} \geq 0$. For all real $k=\left(k_{x}, k_{y}\right)$, the matrix $Q_{k}$ is positive definite since for $r>0$ all eigenvalues

$$
\lambda_{1,2}=1, \quad \lambda_{3,4}=|k|^{2}+\gamma \pm k_{x} k_{y}, \quad \lambda_{5,6}=\frac{1}{2}\left(1+|k|^{2}+\gamma \pm \sqrt{\left(1+|k|^{2}+\gamma\right)^{2}-4 \gamma}\right)
$$

are strictly positive. Hence there is a constant $c_{2}>0$ such that pointwise a.e.

$$
\overline{\widetilde{u}}^{T} Q_{k} \tilde{u} \geq c_{2} \overline{\widetilde{u}}^{T} \tilde{u},
$$


and by the definition of the scalar product (4.6) we arrive at

$$
\left\langle\widehat{u} \mid S_{\gamma} \widehat{u}\right\rangle_{H^{1}} \geq c_{1} c_{2} \int_{-H}^{+H} \overline{\widetilde{u}}^{T} \tilde{u} d z=c_{1} c_{2}\|\widehat{u}\|_{H^{1}}^{2}
$$

Now we can apply Proposition 2.4 to the pair $M, S_{\gamma}$ and get the following.

Proposition 4.2. There exists an increasing sequence $0 \leq \omega_{n}^{2} \rightarrow \infty$ and a sequence $\widehat{u}_{n} \in H^{1}$ such that

$$
\begin{gathered}
\omega_{n}^{2} M \widehat{u}_{n}=S_{k} \widehat{u}_{n} \\
\left(\gamma+\omega_{n}^{2}\right)\left\langle\widehat{u}_{n} \mid M \widehat{u}_{m}\right\rangle_{H^{1}}=\delta_{n, m}=\frac{\gamma+\omega_{n}^{2}}{\omega_{n}^{2}}\left\langle\widehat{u}_{n} \mid S_{k} \widehat{u}_{m}\right\rangle_{H^{1}},
\end{gathered}
$$

and each $\widehat{u} \in H^{1}$ can be uniquely represented by an $H^{1}$-convergent series

$$
\widehat{u}=\sum_{n=0}^{\infty} \alpha_{n} \widehat{u}_{n}
$$

with an $\ell_{2}$-sequence $\alpha_{n}=\left(\left(\gamma+\omega_{n}^{2}\right) / \omega_{n}^{2}\right)\left\langle\widehat{u} \mid S_{k} \widehat{u}_{n}\right\rangle_{H^{1}}$.

Proof. Let the assertions of Proposition 2.4 appropriately hold for the pair $M, S_{\gamma}$. At first we observe that by (2.10) we have

$$
\lambda_{1}=\frac{\left\langle\widehat{u}_{1} \mid S_{\gamma} \widehat{u}_{1}\right\rangle_{H^{1}}}{\left\langle\widehat{u}_{1} \mid M \widehat{u}_{1}\right\rangle_{H^{1}}}=\gamma+\frac{\left\langle\widehat{u}_{1} \mid S_{k} \widehat{u}_{1}\right\rangle_{H^{1}}}{\left\langle\widehat{u}_{1} \mid M \widehat{u}_{1}\right\rangle_{H^{1}}} \geq \gamma,
$$

and hence $\omega_{n}^{2}:=\lambda_{n}-\gamma \geq 0$ and $\omega_{n}^{2} \rightarrow \infty$ increasingly. By (2.10), on one hand, we then trivially have

$$
\left(\gamma+\omega_{n}^{2}\right)\left\langle\widehat{u}_{n} \mid M \widehat{u}_{m}\right\rangle_{H^{1}}=\lambda_{n}\left\langle\widehat{u}_{n} \mid M \widehat{u}_{m}\right\rangle_{H^{1}}=\delta_{n, m}
$$

and, on the other hand, we further conclude that

$$
\delta_{n, m}=\left\langle\widehat{u}_{n} \mid S_{\gamma} \widehat{u}_{m}\right\rangle_{H^{1}}=\gamma\left\langle\widehat{u}_{n} \mid M \widehat{u}_{m}\right\rangle_{H^{1}}+\left\langle\widehat{u}_{n} \mid S_{k} \widehat{u}_{m}\right\rangle_{H^{1}}=\frac{\gamma}{\gamma+\omega_{n}^{2}} \delta_{n, m}+\left\langle\widehat{u}_{n} \mid S_{k} \widehat{u}_{m}\right\rangle_{H^{1}} .
$$

Finally each $\widehat{u} \in H^{1}$ can be uniquely represented by an $H^{1}$-convergent series

$$
\widehat{u}=\sum_{n=0}^{\infty} \alpha_{n} \widehat{u}_{n}
$$


with an $\ell_{2}$-sequence $\alpha_{n}=\left\langle\widehat{u} \mid S_{\gamma} \widehat{u}_{n}\right\rangle_{H^{1}}$. Evaluating the scalar product of both sides of the above equation with $S_{k} \widehat{u}_{m}$, we get

$$
\left\langle\widehat{u} \mid S_{k} \widehat{u}_{m}\right\rangle_{H^{1}}=\sum_{n=0}^{\infty} \alpha_{n}\left\langle\widehat{u}_{n} \mid S_{k} \widehat{u}_{m}\right\rangle_{H^{1}}=\alpha_{m} \frac{\omega_{m}^{2}}{\gamma+\omega_{m}^{2}} .
$$

The next proposition together with Proposition 4.1 finally shows that all these weak $H^{1}$-solutions are indeed strong solutions.

Proposition 4.3. If $\widehat{u} \in H^{1}$ fulfills (4.2) for all $\widehat{v} \in H^{1}$, then $\widehat{u}$ is continuous and layerwise $\mathcal{C}^{\infty}$.

Proof. We inductively show that the distributional derivatives of $\widehat{u}$ can be identified with smooth functions. Expanding the operator $L$ according to its definition and rearranging (4.2) give

$$
\begin{aligned}
\int_{-H}^{+H} \bar{\partial}_{z} \widehat{v}^{T} L_{z}^{T} C L_{z} \partial_{z} \widehat{u} d z= & \int_{-H}^{+H} \overline{\widehat{v}}^{T}\left(\omega^{2} \rho+\left(i k_{x} L_{x}+i k_{y} L_{y}\right)^{T} C L_{k}\right) \widehat{u} d z \\
& -\int_{-H}^{+H} \bar{\partial}_{z}^{T} L_{z}^{T} C\left(i k_{x} L_{x}+i k_{y} L_{y}\right) \widehat{u} d z .
\end{aligned}
$$

We fix a layer $l$ and take an arbitrary $\mathcal{C}^{\infty}$-function $\widehat{v}$ with compact support in $\left(H_{l-1}, H_{l}\right)$. As $C_{l}$ is symmetric, for those $\widehat{v}$ the left-hand side can be written as

$$
\int_{H_{l-1}}^{H_{l}}{\overline{\partial_{z}\left(L_{z}^{T} C_{l} L_{z}\right)}}_{\widehat{v}}^{T} \partial_{z} \widehat{u} d z .
$$

We integrate by parts on the right-hand side to get

$$
\begin{aligned}
\int_{H_{l-1}}^{H_{l}} \overline{\partial_{z}\left(L_{z}^{T} C_{l} L_{z}\right) \widehat{v}} \partial_{z} \widehat{u} d z= & \int_{H_{l-1}}^{H_{l}} \overline{\widehat{v}}^{T}\left(\omega^{2} \rho+\left(i k_{x} L_{x}+i k_{y} L_{y}\right)^{T} C_{l} L_{k}\right) \widehat{u} d z \\
& +\int_{H_{l-1}}^{H_{l}} \overline{\widehat{v}}^{T} L_{z}^{T} C_{l}\left(i k_{x} L_{x}+i k_{y} L_{y}\right) \partial_{z} \widehat{u} d z .
\end{aligned}
$$

The operator $L_{z}^{T} C_{l} L_{z}$ is invertible since $C_{l}$ is positive definite and $L_{z}$ is injective; hence the previous equation for all $\widehat{v}$ is equivalent to

$$
\int_{H_{l-1}}^{H_{l}} \bar{\partial}_{z} \widehat{v}^{T} \partial_{z} \widehat{u} d z=-\int_{H_{l-1}}^{H_{l}} \overline{\widehat{v}}^{T} A_{l} \widehat{u} d z,
$$

where

$$
A_{l} \widehat{u}:=-\left(L_{z}^{T} C_{l} L_{z}\right)^{-1}\left(\omega^{2} \rho+\left(i k_{x} L_{x}+i k_{y} L_{y}\right)^{T} C_{l} L_{k}+L_{z}^{T} C_{l}\left(i k_{x} L_{x}+i k_{y} L_{y}\right) \partial_{z}\right) \widehat{u} .
$$



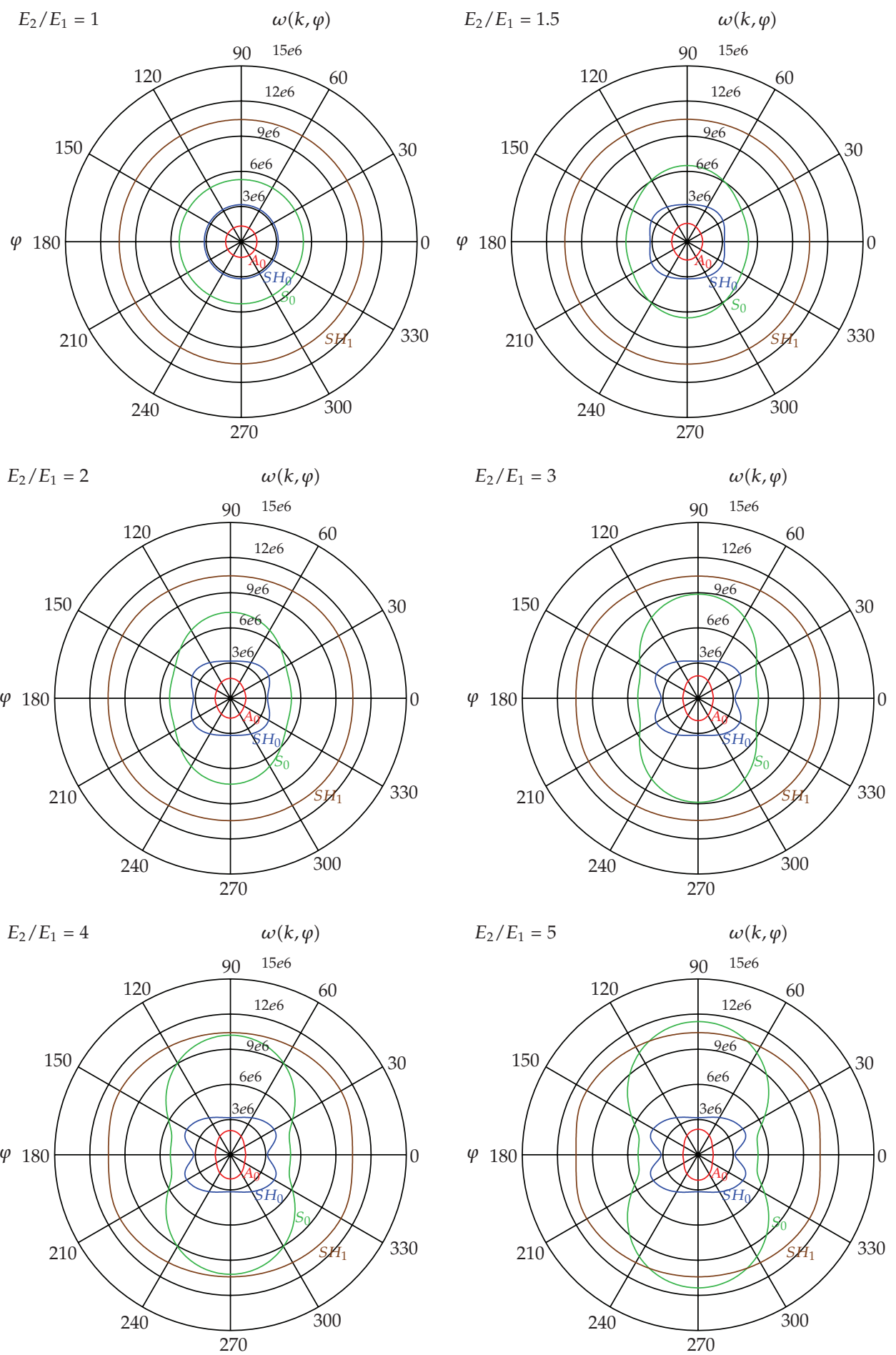

Figure 2: Direction dependence of circular frequency $\omega(k, \phi)\left[\mathrm{s}^{-1}\right]$ at circular wavenumber $k=1000 \mathrm{~m}^{-1}$ for different ratios $E_{2} / E_{1}$. As one can see, each mode has its own individual anisotropic behaviour; even the sequence of the wave modes ordered by their frequencies is angle dependant. 

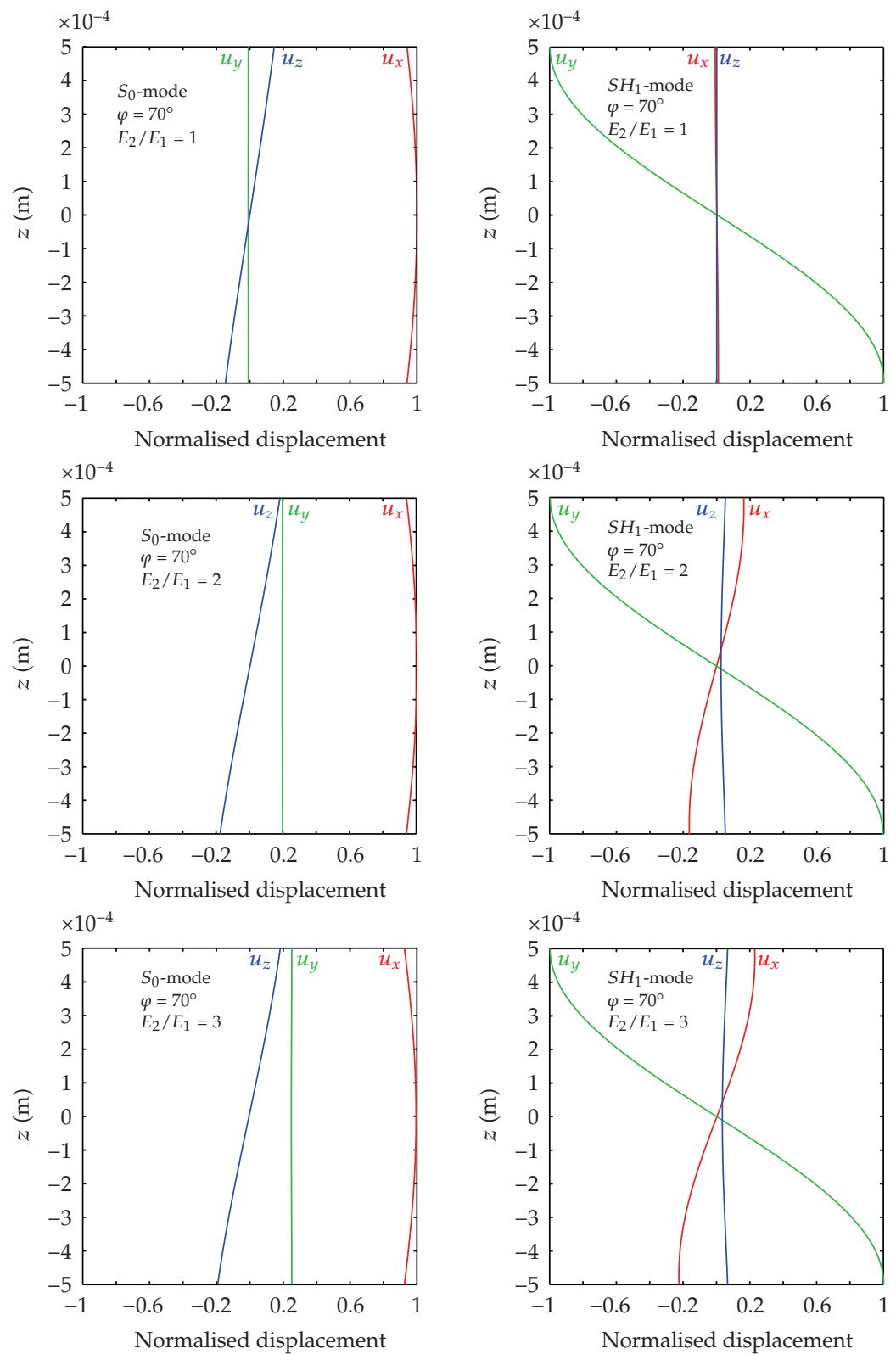

Figure 3: Components of the displacement vector $u=\left(u_{x}, u_{y}, u_{z}\right)$ over the thickness of the plate at $\phi=70^{\circ}$ of $S_{0}$ and $S H_{1}$-modes for different ratios $E_{2} / E_{1}$. Here $u_{x}$ and $u_{y}$ are the in-plane components in longitudinal and transversal directions, respectively. Obviously the distinction between shear-horizontal and in-plane Lamb-waves becomes less clear in an anisotropic material. This means especially that common measuring techniques quantifying the $z$ component will detect also the $S H$-modes.

Equation (4.31) states that the second distributional derivative $\left.\partial_{z}^{2} \widehat{u}\right|_{\left(H_{l-1}, H_{l}\right)}$ of $\widehat{u}$ restricted to $\left(H_{l-1}, H_{l}\right)$ can be identified with $\left.A_{l} \widehat{u}\right|_{\left(H_{l-1}, H_{l}\right)}$. Since $\widehat{u} \in H^{1}$, we have $\left.A_{l} \widehat{u}\right|_{\left(H_{l-1}, H_{l}\right)} \in$ $L^{2}\left(H_{l-1}, H_{l}\right)$. Consequently, we may assume that $\left.\widehat{u}\right|_{\left(H_{l-1}, H_{l}\right)} \in H^{2}\left(H_{l-1}, H_{l}\right)$ and hence $\left.\widehat{u}\right|_{\left(H_{l-1}, H_{l}\right)} \in \mathcal{C}^{1}\left(\left[H_{l-1}, H_{l}\right]\right)$. From this we in turn infer that $\left.A_{l} \widehat{u}\right|_{\left(H_{l-1}, H_{l}\right)} \in \mathcal{C}\left(\left[H_{l-1}, H_{l}\right]\right)$, and 
(4.31) ensures that $\left.\partial_{z}^{2} \widehat{u}\right|_{\left(H_{l-1}, H_{l}\right)} \in \mathcal{C}\left(\left[H_{l-1}, H_{l}\right]\right)$; that is, $\left.\widehat{u}\right|_{\left(H_{l-1}, H_{l}\right)} \in \mathcal{C}^{2}\left(\left[H_{l-1}, H_{l}\right]\right)$. Repeating this argument proves the assertion.

We conclude this section by deriving a mode decomposition of general waves travelling in the plate. For a function $u=u(x, y)$, we denote by $\widehat{u}=\widehat{u}\left(k_{x}, k_{y}\right)$ its Fourier transform in the wavenumber domain

$$
\widehat{u}\left(k_{x}, k_{y}\right)=\frac{1}{(2 \pi)^{2}} \iint_{\mathbb{R}^{2}} u(x, y) e^{-i\left(k_{x} x+k_{y} y\right)} d x d y .
$$

Let again $u=\left(u_{x}, u_{y}, u_{z}\right)^{T}=u(x, y, z, t)$ be the displacement vector of a wave travelling in the plate in the absence of external forces (e.g., as a result of an initial excitation which now has stopped) such that it fulfills (3.9), (3.10), and (3.11). By formally applying the Fourier transform with respect to $(x, y)$ to these equations, we see that $\widehat{u}=\widehat{u}(k, z, t)=\widehat{u}\left(k_{x}, k_{y}, z, t\right)$ then fulfills

$$
\rho_{l} \ddot{\hat{u}}=L_{k}^{T} C_{l} L_{k} \widehat{u}, \quad \forall z \in\left(H_{l-1}, H_{l}\right), l=1, \ldots, N
$$

and the boundary and interface conditions (3.14) and (3.15). Suppose that for all $k$ and $t$ (or at least for the ones of interest) the functions $z \mapsto \widehat{u}(k, z, t)$ and $z \mapsto \ddot{\widetilde{u}}(k, z, t)$ are in $H^{1}$. Analogously to the previous considerations, the weak form of (4.34), (3.14), and (3.15) is then equivalent to

$$
M \ddot{\hat{u}}=-S_{k} \widehat{u} .
$$

According to Proposition 4.2 and (2.11), we can represent $\widehat{u}(k, z, t)$ by a series with the eigenvectors $\widehat{u}_{n}(k, z)$ as basis functions

$$
\widehat{u}(k, z, t)=\sum_{n=0}^{\infty} \alpha_{n}(k, t) \widehat{u}_{n}(k, z), \quad \ddot{\widehat{u}}(k, z, t)=\sum_{n=0}^{\infty} \ddot{\alpha}_{n}(k, t) \widehat{u}_{n}(k, z) .
$$

We remark that for different $k$ we have different basis functions $\widehat{u}_{n}=\widehat{u}_{n}(k)$, hence the dependence $\alpha_{n}=\alpha_{n}(k)$; but for fixed $k$ we fix this basis for all times $t$, hence the dependence $\alpha_{n}=\alpha_{n}(k, t)$. Furthermore we assume that $\ddot{\hat{u}}$ can indeed be represented with $\ddot{\alpha}_{n}$; that is, summation and time derivation commute. Applying the orthogonality relations in (4.21) to (4.35), we get for fixed $k$ the decoupled system of ordinary differential equations in $t$ :

$$
\ddot{\alpha}_{n}(k, t) \frac{1}{\gamma+\omega_{n}(k)^{2}}=-\alpha_{n}(k, t) \frac{\omega_{n}(k)^{2}}{\gamma+\omega_{n}(k)^{2}} \Longleftrightarrow \ddot{\alpha}_{n}(k, t)+\omega_{n}(k)^{2} \alpha_{n}(k, t)=0,
$$

with general solutions

$$
\alpha_{n}(k, t)=a_{n}(k) e^{i \omega_{n}(k) t}+b_{n}(k) e^{-i \omega_{n}(k) t} .
$$


Assuming that the following operations are valid, we get the mode decomposition

$$
\begin{aligned}
u(x, y, z, t)= & \iint_{\mathbb{R}^{2}} \widehat{u}\left(k_{x}, k_{y}, z, t\right) e^{i\left(k_{x} x+k_{y} y\right)} d k_{x} d k_{y} \\
= & \sum_{n=0}^{\infty} \iint_{\mathbb{R}^{2}}\left(a_{n}(k) \widehat{u}_{n}(k, z) e^{i \omega_{n}(k) t}+b_{n}(k) \widehat{u}_{n}(k, z) e^{-i \omega_{n}(k) t}\right) \\
& \times e^{i\left(k_{x} x+k_{y} y\right)} d k_{x} d k_{y} .
\end{aligned}
$$

\section{Numerical Results}

The generalized eigenvalue problem can efficiently be solved by a suitable finite-element discretization, leading to a finite-dimensional generalized eigenvalue problem of the same form of (4.10) with mass matrix $M$ and stiffness matrix $S_{k}$; see [7-9]. A comprehensive treatment of the mathematical theory and practice of FEM can be found in the study by Ern and Guermond in [21]. For theoretical results concerning order-preserving convergence of the eigenvalues of the discretized problem to the eigenvalues of the infinite-dimensional problem, we refer to the studies by Yang and Chen in [22].

As an application we want to examine how dispersion curves vary with an increasing degree of anisotropy. To do so, we consider a transversely isotropic material with $y$-axis as the axis of rotational symmetry. We note that unidirectional fibre-reinforced composites can often be approximately modelled by transversely isotropic material so that symmetry-axis and fibre direction coincide. For a description of the elastic material constants in this case we refer to the studies by Ambartsumyan in [23] and Altenbach et al. in [24]. We used as Young's moduli $E_{1}=E_{3}=70 \mathrm{GPa}, E_{2}=\theta E_{1}$, as Poisson's ratios $v_{32}=v_{31}=v_{13}=v_{12}=0.3$, $v_{23}=v_{21}=v_{32} / \theta$, and as shear moduli $G_{23}=G_{12}=G_{13}=E_{1} /\left(2\left(1+v_{23}\right)\right)$, where the ratio $\theta=E_{2} / E_{1}$ determines the degree of anisotropy. The material behaves isotropic for $\theta=1$ while for $\theta>1$ it becomes transversely isotropic with rotational symmetry around the $y$-axis. In this case the matrix $C$ of material constants can be written as

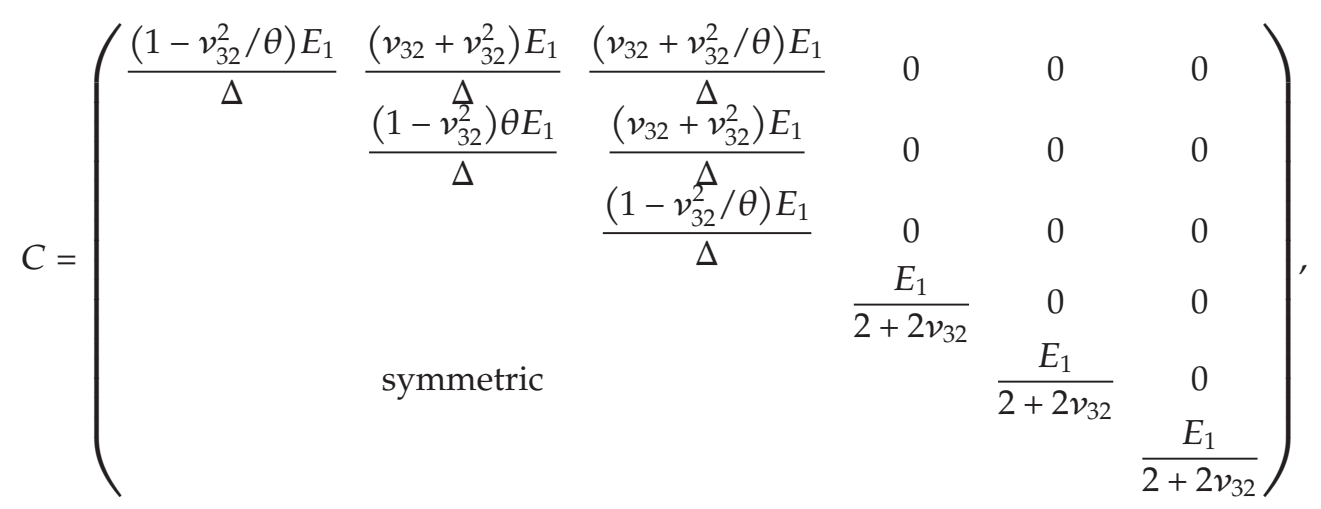

with

$$
\Delta:=1-\left(\frac{2}{\theta}+1\right) v_{32}^{2}-\frac{2}{\theta} v_{32}^{3}
$$


The plate is assumed to have a thickness of $d=1 \mathrm{~mm}$ and a mass density of $\rho=$ $2700 \mathrm{~kg} \cdot \mathrm{m}^{-3}$. We computed the circular frequency $\omega(k, \phi)$ for a fixed circular wavenumber in dependence of the propagation direction $\phi$ and for different ratios $\theta=E_{2} / E_{1}$. Some results for the first four modes are displayed in Figure 2 which illustrate the increasingly anisotropic behaviour. In the strictly transversely isotropic case $\theta>1$, we labeled the modes according to their isotropic counterparts, but it becomes clear from Figure 3 that the distinction between pure $S H$ - and $S$-modes is growing less obvious as both kinds of modes get components in all directions.

\section{Acknowledgment}

The work of the authors is being supported by Deutsche Forschungsgemeinschaft (DFG) under Schu 1978/4-1.

\section{References}

[1] V. Giurgiutiu, Structural Health Monitoring with Piezoelectric Wafer Active Sensors, Academic Press, New York, NY, USA, 2008.

[2] J. Rose, Ultrasonic Waves in Solid Media, Cambridge University Press, Cambridge, UK, 1999.

[3] J. D. Achenbach, Wave Propagation in Elastic Solids, North-Holland, Amsterdam, The Netherlands, 1973.

[4] M. J. S. Lowe, "Matrix techniques for modeling ultrasonic waves in multilayered media," IEEE Transactions on Ultrasonics, Ferroelectrics, and Frequency Control, vol. 42, no. 4, pp. 525-542, 1995.

[5] S. B. Dong and R. B. Nelson, "On natural vibrations and waves in laminated orthotropic plates," Journal of Applied Mechanics, vol. 39, no. 3, pp. 739-745, 1972.

[6] B. Aalami, "Waves in prismatic guides of arbitrary cross section," Journal of Applied Mechanics, vol. 40, no. 4, pp. 1067-1072, 1973.

[7] E. Kausel, "Wave propagation in anisotropic layered media," International Journal for Numerical Methods in Engineering, vol. 23, no. 8, pp. 1567-1578, 1986.

[8] J. M. Galán and R. Abascal, "Numerical simulation of Lamb wave scattering in semi-infinite plates," International Journal for Numerical Methods in Engineering, vol. 53, no. 5, pp. 1145-1173, 2002.

[9] G. R. Liu and Z. C. Xi, Elastic Waves in Anisotropic Laminates, CRC Press, Boca Raton, Fla, USA, 2002.

[10] L. Gavrić, "Computation of propagative waves in free rail using a finite element technique," Journal of Sound and Vibration, vol. 185, no. 3, pp. 531-543, 1995.

[11] I. Bartoli, A. Marzani, F. Lanza di Scalea, and E. Viola, "Modeling wave propagation in damped waveguides of arbitrary cross-section," Journal of Sound and Vibration, vol. 295, no. 3-5, pp. 685-707, 2006.

[12] A. Marzani, E. Viola, I. Bartoli, F. Lanza di Scalea, and P. Rizzo, "A semi-analytical finite element formulation for modeling stress wave propagation in axisymmetric damped waveguides," Journal of Sound and Vibration, vol. 318, no. 3, pp. 488-505, 2008.

[13] F. Treyssède, "A semi-analytical finite element method for elastic guided waves propagating in helical structures," The Journal of the Acoustical Society of America, vol. 123, no. 5, p. 3841, 2008.

[14] T. Bouhennache, "Spectral analysis of an isotropic stratified elastic strip and applications," Osaka Journal of Mathematics, vol. 37, no. 3, pp. 577-601, 2000.

[15] P. D. Lax, Functional Analysis, Pure and Applied Mathematics, Wiley-Interscience, New York, NY, USA, 2002.

[16] J. B. Conway, A Course in Functional Analysis, vol. 96 of Graduate Texts in Mathematics, Springer, New York, NY, USA, 1985.

[17] H. Schröder, Funktionalanalysis, Harri Deutsch, Frankfurt am Main, Thun, Switzerland, 2nd edition, 2000.

[18] J. E. Marsden and T. J. R. Hughes, Mathematical Foundations of Elasticity, Dover, New York, NY, USA, 1994.

[19] R. A. Adams, Sobolev Spaces, vol. 6 of Pure and Applied Mathematics, Academic Press, New York, NY, 
USA, 1975.

[20] V. G. Maz'ja, Sobolev Spaces, Springer Series in Soviet Mathematics, Springer, Berlin, Germany, 1985.

[21] A. Ern and J.-L. Guermond, Theory and Practice of Finite Elements, vol. 159 of Applied Mathematical Sciences, Springer, New York, NY, USA, 2004.

[22] Y. Yang and Z. Chen, "The order-preserving convergence for spectral approximation of self-adjoint completely continuous operators," Science in China. Series A, vol. 51, no. 7, pp. 1232-1242, 2008.

[23] S. A. Ambartsumyan, Theory of Anisotropic Plates: Strength, Stability, and Vibrations, Hemisphere, New York, NY, USA, 1991.

[24] H. Altenbach, J. Altenbach, and R. Rikards, Einführung in die Mechanik der Laminat-und Sandwichtragwerke, Deutscher Verlag für Grundstoffindustrie, Stuttgart, Germany, 1996. 


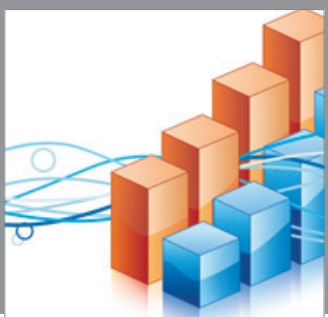

Advances in

Operations Research

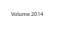

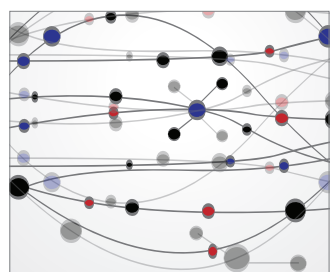

\section{The Scientific} World Journal
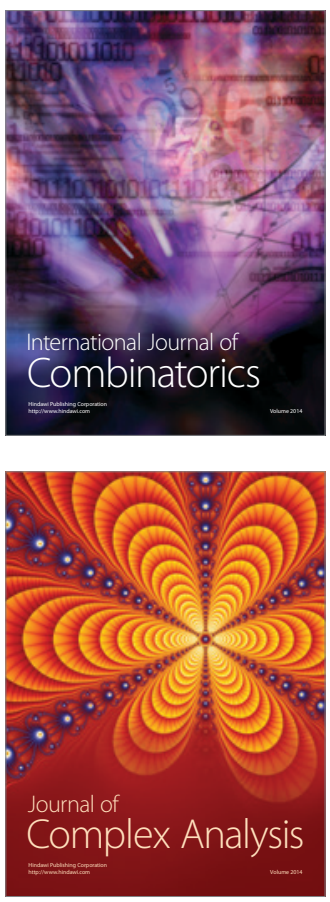

International Journal of

Mathematics and

Mathematical

Sciences
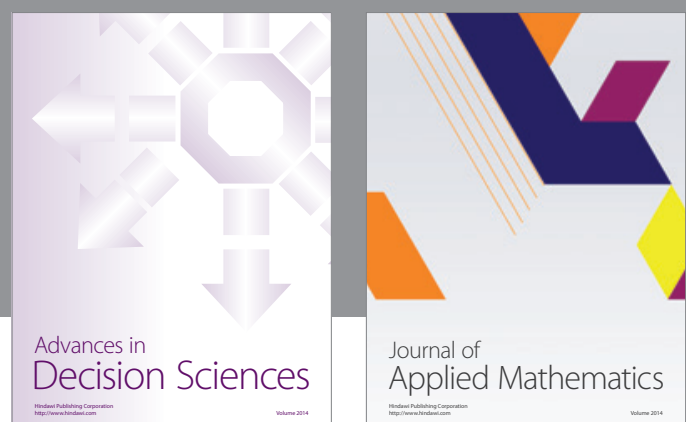

Journal of

Applied Mathematics
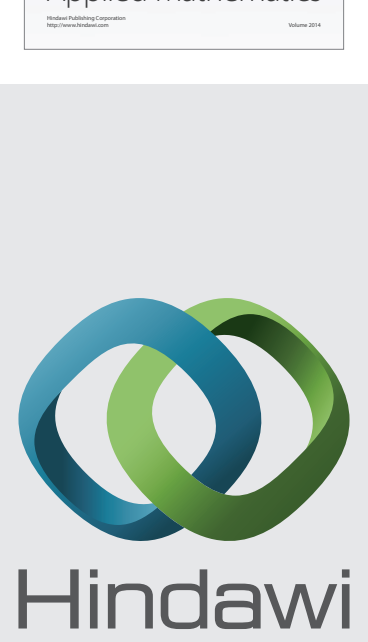

Submit your manuscripts at http://www.hindawi.com
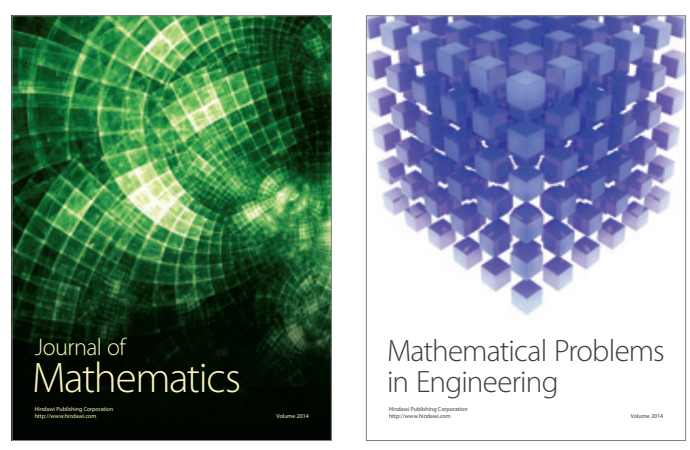

Mathematical Problems in Engineering
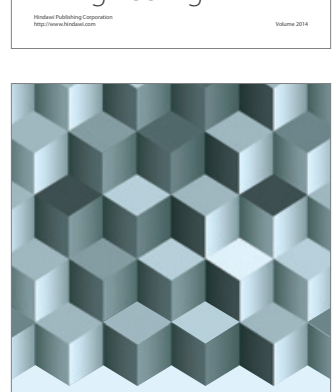

Journal of

Function Spaces
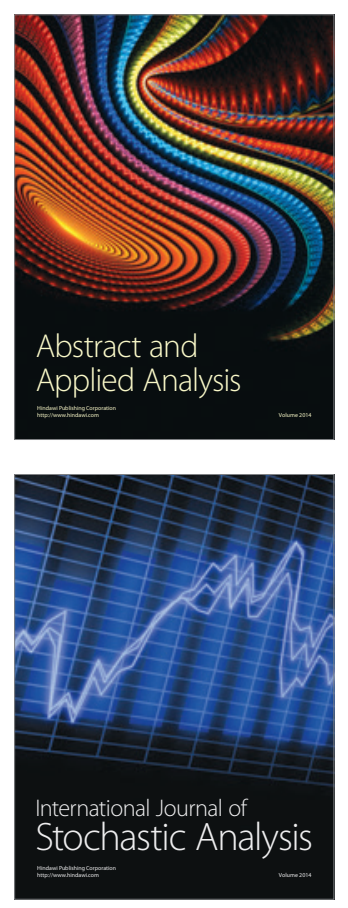

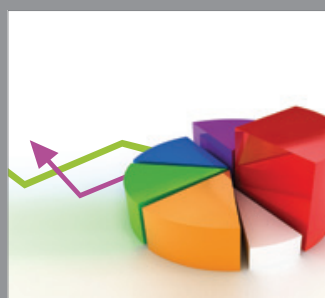

ournal of

Probability and Statistics

Promensencen
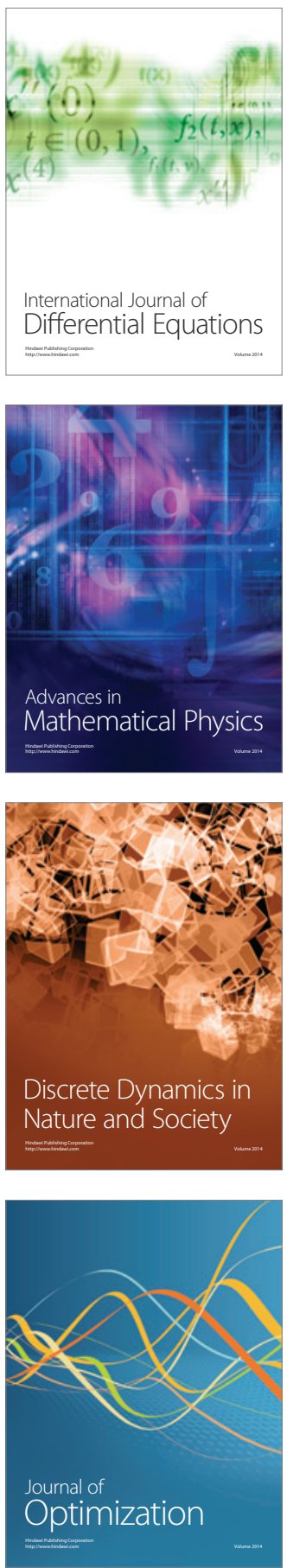